\title{
Preliminary table of authorities
}

\section{NATIONAL CASES}

\section{Argentina}

Chevron case, Supreme Court 2013 84

\author{
Australia \\ CSR Ltd v. Wren (1997) 44 NSWLR 463 \\ Dagi v. B.H.P (No 2) [1997] 1 VR 428 \\ 4,175 \\ James Hardie \& Co Pty Ltd v. Hall (1998) 43 NSWLR 554 \\ Voth v. Manildra Flour Mill (1990) 171 CLR 538 (HCA) \\ Zhang v. Zemin [2010] 243 FLR 299

\section{Belgium}

Burmese Refugees case, Cour de Cassation 2005, 2007

\section{Canada}

Anvil Mining Ltd. v. Canadian Ass'n Against Impunity [2012] CA 117, para 81

(Can Que)

Bellavance v. Blais, [1976] RP 423 (CA) ...................................................... 207

Bil'in (Village Council) v. Green Park Int'l Ltd 2009 QCCS 4151; [2010] CA 1455;

[2011] 1 SCR vi (dismissing application with costs)

$5,41,206,207$

Bouzari v. Islamic Republic of Iran [2002] OJ No 1624; [2004] OJ No. 2800 Docket No. C38295

Bouzari v. Islamic Republic of Iran, (2004), 71 OR (3d) 675 (Ont CA); leave to appeal refused, [2005] 1 SCR vi

Chevron Corp. v. Yaiguaje (SCC 35682) 84,85

Choc v. Hudbay Minerals Inc, 2013 Carswell Ont 10514 $4,188,249$

Gouvernement de la République démocratique du Congo v. Venne, [1971] SCR 997

Désiré Munyaneza case, 1994

Edward Tracy v. Iranian Ministry of Information and Security 2014 ONSC 1696 (CanLII)

Mack v. Canada (Attorney General), (2002), 60 OR (3d) 737 (CA), leave to appeal refused, [2003] 1 SCR xiii

R v. Hape [2007] SCC 26; [2007] 1 SCR 292 206

Recherches Internationales Quebec v. Cambior Inc, [1998] QJ No 2554, 14 August 1998 4, 175

Reference re Newfoundland Continental Shelf, [1984] 1 SCR 86 205 
Reference re Secession of Quebec, [1998] 2 SCR 217 205

Suresh v. Canada (Minister of Citizenship and Immigration), [2002] 1 SCR 3; 2002 SCC 1

\section{Ecuador}

Aguinda v. Chevron Corp, No. 002-2003 (Super Ct of Nueva Loja, Feb. 14, 2011);

Ct of Cassation 2003 $83,84,86$

\section{France}

Association France-Palestine Solidarite 'AFPS' vs Societe Alstom Transport SA, Versailles CA 209,249

Burmese Refugees case, Tribunal de Grande Instance Nanterre March 10, 2006

\section{Germany}

Prosecutor v. Jorgic, Bundesverfassungsgericht (Federal Constitutional Court), 2 BvR 1290/99 (Decision of December 12, 2000), reprinted in Neue Juristische Wochenschrift 1848, 1852 (2001)

\section{India}

Bhopal litigation (Union Carbide)

\section{Ireland}

Summers Jennings v. Furlong, [1966] IR 183, 186, 190 ................................ 205

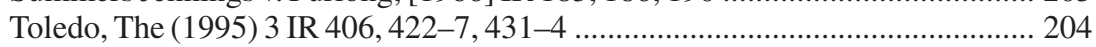

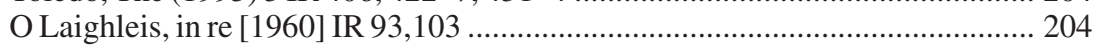

\section{Israel}

Attorney Gen'l of Israel v. Eichmann, 36 ILR 277, 303-4 (Isr S Ct, 1962) ...... 15, 31

\section{Italy}

Ferrini v. Federal Republic of Germany, 11 March 2004, 128 ILR 659

\section{Netherlands}

Al Haq, Criminal Complaint Lodged Against Dutch Company for Construction of Settlements and 'The Wall' (Oct. 14, 2010)

Ashraf Ahmed El-Hojouj v. Harb Amer Derbal et al Hague District Court, No. 400882 (Mar. 21, 2012)

BATCO, Amsterdam Commerce Chamber of the Court of Amsterdam CA 21 June 1979, (NJ 1980, 71)

Chevron case, Supreme Court Sept 262014

Kouwenhoven case, District Court of The Hague, 7 June 2006, Case No. AY516;

Supreme Court, 20 April 2010, Case No BK8132 .... 38,39

Ogoru v. Royal Dutch Shell, C/09/330891 / HA ZA 09-0579

Ogoru v. Shell Petroleum NV, C/09/365498 HA ZA 10-167 ..... 
Van Anraat case, District Court of The Hague, 23 December 2005, Case No. AX6406; Court of Appeal of The Hague, 9 May 2007, Case No. BA6734; Supreme Court, 30 June 2009, Case No. BG4822

\section{Switzerland}

ECCHR case against Nestle

TRIAL case against Argor-Heraeus

\section{United Kingdom}

Adams v. Cape Industries plc [1990] Ch 433 CA 180,182

Al Adsani v. Kuwait (1995) 100 ILR 465, The Times 29 March 1996, 103 ILR 420 (QB 1995), aff'd 107 ILR 536 (CA.1996) ..... 5, 41, 45, 68, 175, 179, 199, 201,

Belhaj v. Straw [2014] EWCA Civ 1394 $177,179,201$

Bodo Community, Claim No. HQ 11X01280, High Court (QB) 43

Bodo Community v. Shell Petroleum Development Company of Nigeria Ltd [2014] EWHC 197 (TCC) 191,192

Boys v. Chaplin [1971] AC 356 190

Chandler (David Brian) v. Cape plc [2012] EWCA Civ 525 ....... 185, 188, 189, 233

Colt Indus. v. Sarlie [1966] 1 WLR 440 (CA) 42

Connelly v. RTZ Corporation plc and Ritz Overseas Ltd, [1998] AC 854, HL ... 174, $183,185,190,192,194$

Coupland v. Arabian Gulf Petroleum Co [1983] 3 All ER 226, CA 190

DHN v. London Borough of Tower Hamlets [1976] 1 WLR 852, CA 90,180

Fish \& Fish Ltd v. Sea Shepherd UK (The Steve Irwin) [2013] EWCA Civ 544; [2013] 3 All ER 867 202 Guerrero v. Monterrico Metals plc [2009] EWHC 2475(QB) ........ 4, 175, 186, 191 Harding v. Wealands [2006] UKHL 32; [2006] 3 WLR 83 193, 194 Harrods (Buenos Aires), Re [1992] Ch 72, CA ................................................ 174 Hutchinson v. Newbury Magistrates Court (2000) ILR 499 .............................. 197 Jones v. Saudi Arabia [2006] UKHL 26, [2006] 2 WLR 1424; [2007] 1 AC 270 .... 5, $41,45,68,179,199,201,202$

Kesabo v. African Barrick Gold plc [2013] EWHC 4045 (QB) 176

Khurts Bat v. Investigating Judge of the German Federal Court [2011] EWHC 2029 (Admin)

Kuwait Airways Corp v. Iraqi Airways Co (No 5) [2002] UKHL 19; [2002] 2 AC 883

Kuwait Airways Corp v. Iraqi Airways (Nos 4 and 5) [2002] UKHL 19; [2002] 2 AC 886

Lennard's Carrying Co Ltd v. Asiatic Petroleum Co Ltd [1915] AC 705 ................ 35

Lubbe v. Cape plc [2000] UKHL 41 ............................................................ 3, 4

Lubbe v. Cape plc (No. 2) [2000] 1 WLR 1545 (HL) ........... 3, 4, 174, 184, 190, 194

Maclaine Watson v. Dept of Trade and Industry [1989] Ch 72 (CA) .................. 198

Maharanee of Baroda v. Wildenstein (1972) 2 QB 283 ..................................... 42

Meridian Global Funds Management Asia Ltd v. Securities Commission [1995] 2 $\mathrm{AC} 500$ (PC) ....................................................................................... 35

Metall und Rohstoff v. Donaldson Lufkin \& Jenrette Inc [1990] 1 QB 391 ......... 190 
Mostyn v. Fabrigas (1775) 1 Cowper 161

Motto \& Ors v. Trafigura [2011] EWHC 90206

Ngcobo v. Thor Chemicals Ltd, The Times, Nov 10 1995, CA .... 174, 183, 185, 190

Okura \& Co Ltd v. Forsbacka Jernverks Aktiebolag [1914] 1 KB 715, 721 ......... 182

Oppenheimer v. Cattermole (Inspector of Taxes) [1976] AC 249, 278 ................ 178

Oppenheimer v. Louis Rosenthal \& Co. AG [1937] 1 All ER 23 ........................... 43

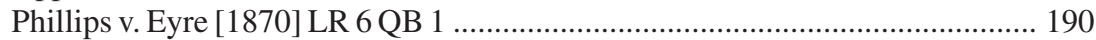

Prest v. Petrodel [2013] UKSC 34; [2013] 2 AC 415 ........................................... 182

Propend Finance Pty v. Sing (1997) 111 ILR 611 (CA) ...................................... 199

R v. Bow Street Magistrates; ex parte Pinochet Ugarte (No 3) [1999] 2 WLR 827;

[2000] 1 AC 147

$27,31,33,196,200$

R v. British Steel plc [1995] 1 WLR 1356 ..................................................... 35

R v. Jones (Margaret) [2006] UKHL 16; [2007] 1 AC 136 ................................ 197

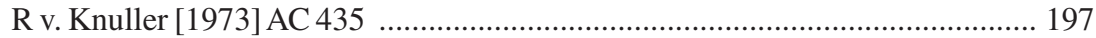

Red Sea Insurance Co Ltd v. Bouygues SA [1994] 3 All ER 749 ....................... 190

Salomon v. Salomon [1897] AC 22 .............................................................. 180

Sea Assets v. PT Garuda Indonesia (2000) 4 All ER 371 .................................... 42

Sithole v. Thor Chemicals Ltd, 2000 WL 1421183 (CA) ......................... 174, 183

Smith v. Littlewoods Ltd [1987] AC 241, 270 ............................................... 185

South India Shipping Corp. v. Export-Import Bank of Korea [1985] 1 WLR

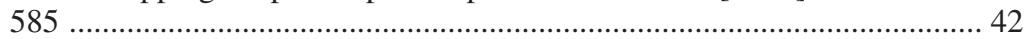

Spiliada Maritime Corp v. Cansulex [1987] AC 460 ............................................ 174

Tesco v. Nattrass [1972] AC 153 ...................................................................... 35

Thompson v. Renwick Group Ltd [2014] EWCA Civ 635 ................................ 188

Trendtex Trading Corp v. Central Bank of Nigeria [1977] QB 529 .................... 197

Vishva Ajay [1989] 2 Lloyd's Rep 558 (QB) ........................................ 43, 177

VTB Capital plc v. Nutritek International Corp [2013] UKSC 5; [2013] 2 AC

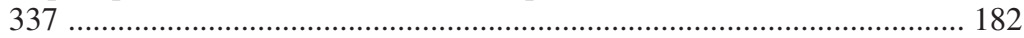

West Rand Central Gold Mining Company v. R [1905] 2 KB 391 ....................... 204

Woolfson v. Strathclyde Regional Council [1978] 38 P\&CR 521 ........................ 180

Young v. Anglo-American [2014] EWCA Civ 1130 ......................................... 173

\section{United States of America}

Abagninin v AMVAC Chemical Corporation 545 F 3d 733 (9th Cir 2008) ......... 124

Abdullahi v Pfizer 562 F 3d 163 (2d Cir 2009) .................................. 121, 126, 151

Abecassis v. Wyatt, 2010 WL 1286871 (SD Tex) ............................................... 129

Adickes v. S H Kress \& Co, 398 US 144, 152, (1970) ........................................ 125

Adikhari v. Daoud and Partners, 2009 WL 6067064 (SD Tex); 2010 WL 744237 (SD Tex)

Aguinda v. Texaco Inc, 142 F Supp 2d 534 (SDNY 2001), aff'd 303 F 3d 470 (2d Cir 2002) $62,64,83-86,93,152$

Al-Aulaqi v.Obama 727 F Supp 2d 1 (DDC 2010) 68,122

Al Shimari v. CACI Int'1, Inc, 658 F 3d 413, 415 (4th Cir 2011), 679 F 3d 205, 212 (4th Cir 2012) ......

Al Shimari v. CACI Intern, Inc 951 F Supp 2d 857 (ED Va 2013) 107,108

Aldana v. Del Monte Fresh Produce, N.A., Inc, 416 F 3d 1242, 1250-51 (11th Cir 2005) $112,120,126$ 
Aldana v. Del Monte Fresh Produce, 578 F 3d 1283 (11th Cir 2009) .................... 66

Alfred Dunhill of London Inc v. Republic of Cuba, 425 US 682 (1976) .............. 198

Ali v. Rumsfeld, 649 F 3d 762 (DC Cir. 2011) ................................................. 106

Allaithi v. Rumsfeld, 753 F 3d 1327 (CADC 2014) ........................................... 70

American Insurance Association v. Garamendi, 539 US 396 (2003) ..................... 76

American Petroleum Institute v. Securities and Exchange Commission 953 F Supp 2d 5 (DDC 2013)

Amlon Metals v. FMC, 775 F Supp 668 (SNDY 1991) ............................. 94, 118

Amoco Cadiz, The [1984] 2 Lloyd's Rep 304, aff'd 954 F 2d 2179 (7th Cir 1992)

160,161

Argentine Republic v. Amerada Hess Shipping Corp, 488 US 428 (1989), 109 S Ct 683

45,95

Ashcroft v. Iqbal, 556 US at 677-8 (2009)

$92,109,252$

Aziz v. Alcolac 658 F 3d 388 (4th Cir 2011) 140

Baker v. Carr, 369 US 186, 210, 82 S Ct 691, 7 L Ed 2d 663 (1962)

73,75

Balintulo v. Daimler AG 727 F 3d 174 (2d Cir 2013) $105,107,108$

Bank of Credit and Commerce International (Overseas) Ltd v. State Bank of Pakistan, 273 F 3d 242, 246 (2d Cir 2001)

Beanal v. Freeport McMoran, Doe I v. Exxon Mobil Corp, 393 F Supp 2d 20 (DDC 2005)

Bell Atlantic Corp. v.Twombly, 550 US 544, 570 (2007) ......................... 92, 93

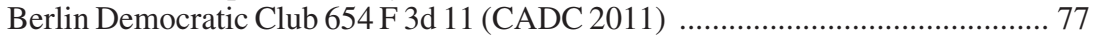

Blackmer v. United States, 284 US 421, 437 (1932) ............................................. 44

Bodner v. Banque Paribas 114 F. Supp 2d 117, 127 (EDNY 2000) ..................... 110

Boim v. Holy Land Foundation 549 F 3d 685 (7th Cir 2008) ............................. 114

Boim v. Quranic Literacy Inst, 291 F 3d 1000, 1012-16 (7th Cir 2002) .............. 115

Boumediene v. Bush, US 128 S Ct 2229, 2262, (2008) ........................................ 77

Bowoto v. Chevron, 312 F Supp 2d 1229 (ND Cal 2004) .... 155-156, 158, 159, 160,

Bowoto v. Chevron Corp, 2006 WL 2455752 (ND Cal.) ............. 124, 126, 130, 132

Bowoto v. Chevron Corp, 2007 WL 2349341 (ND Cal 2007), August 14, 2007

Bowoto v. Chevron 2009 WL 593872 (ND Cal), 3, 9 March 2009 ..................... 150

Bowoto v. Chevron, 2010621 F 3d 1116 (9th Cir 2010) . 95, 112, 129, 142, 147, 167

Boyle v. United Technologies Corporation 487 US 500 (1988) ........................... 69

Bradford ................................................................................................. 106

Bradshaw v. Office of the Architect of the Capitol, 856 F Supp 2d 126,134 (DDC 2012)

Burton v. Wilmington Parking Authority, 365 US 715, 725, (1961) .................. 125

Cabello v. Fernández-Larios, 402 F.3d 1148, 1153-4 (11th Cir. 2005) ....... 111, 129

Caci v. Al Shimari 951 F Supp 2d 857 (ED Va 2013) ......................................... 154

Cardona v. Chiquita Brands International Inc, 760 F.3d 1185 (11th Cir. 2014) 105,106

Cardona v. Chiquita Brands International Inc, S Ct US 2015 2015 WL 1757186 (Mem)

Carijano v. Occidental Petroleum Corp 643 F 3d 1216 (9th Cir 2011) 109, 252

Casey v. US Bank Nat'l Ass'n, 127 Cal App. 4th 1138, 1145 (Cal Ct App 2005) 
Central Bank of Denver v. First Interstate Bank, 511 US 164 (1994) .................. 144

Chavez v. Carranza, 413 F Supp 2d 891, 899 (WD Tenn 2005) .......................... 126

Chavez v. Carranza, 559 F 3d 486, 498-9 (6th Cir), cert. denied, 558 US 822

(2009)

Chevron Corp v. Donziger, 768 F Supp 2d 581 (SDNY 2011); overruled Chevron

Corporation v. Naranjo, 667 F 3d 232 (2d Cir 2012) ................................... 84

Chevron Corp. v. Donziger, 974 F Supp 2d 362 (SDNY 2014) ........................... 84

Chowdhury v. Worldtel Bangladesh Holding, Ltd, 588 F Supp 2d 375 (EDNY 2008, 746 F 3d 42 (2nd Cir 2014) $95,109,111,148,149$

Cicippio-Puleo v. Islamic Republic of Iran 353 F 3d 1024, 1032-3 (DC Cir 2004)

Corrie v. Caterpillar Inc 503 F 3d 974 (9th Cir 2007)

Cuba R. Co. v. Crosby, 222 US 473, 479 (1912) .78

Daimler AG v. Baumann 571 US (2014), 134 S Ct 746 $58,149,251$

David P. Coldesina, DDS v. Estate of Simper, 407 F 3d 1126, 1138 (10th Cir 2005)

Delgado v. Shell Oil Co, 890 F Supp 1324, 1362 (SD Tex 1995)

Demjanjuk v. Petrovsky, 776 F.2d 571, 582-3 (6th Cir. 1985), cert. denied, 475 US 1016(1986)

Doe v. Exxon Corporation, 573 F Supp 2d 16 (DDC 2008) ............................... 171

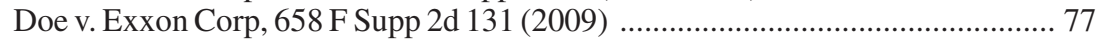

Doe v. Exxon Mobil Corp, 654 F 3d 11, (DC Cir 2011) ............................. 140, 171

Doe v. Exxon Mobil Corp, 527, Fed Appx 7 (CADC July 26 2013) ..... 133, 140, 145

Doe v. Exxon Mobil Corporation, WL 4746256 (DDC) (2014) .............. 55, 71, 108

Doe v. Exxon Corporation 766 F.3d 1013 (9th Cir 2014).................. 140, 167, 243

Doe v. Exxon Corp (Plaintiffs' motion to amend complaint), not reported in

F.Supp.2d, 2 March 2006, WL 516744 (DDC), rev'd on appeal ...... 66-67, 253

Doe v. Nestle, 748 F Supp. 2d 1057 (CD Cal 2010) No. CV 05-5133, slip op. at 120-60 (CD Cal Sept.8 2010)

Doe v. Nestle USA (Inc) F 3d , 2014 WL 4358453 (9th Cir 2014) ............ 108

Doe v. Rumsfeld, 683 F 3d 390, 396 (DC Cir 2012) ........................................... 112

Doe v. Saravia, 348 F Supp 2d 1112, 1150 (E D Cal 2004) ................................ 126

Doe v. Unocal, 67 F Supp 2d 1140, 1147 (CD Cal 1999) ..................................... 168

Doe v. Unocal, 963 F Supp 880, (1997) 27 F Supp 2d 1174 (CD Cal 1998), aff'd 248 F 3d 915 (9th Cir 2001) .... 11, 13, 55, 57, 59, 67, 71, 78, 92, 94, 126, 148, 155,

$167,168,203$

Doe v. Unocal, 110 F Supp 2d 1294 (CD Cal 2000)

Doe v. Unocal (2002), 395 F 3d 932 (9th Cir 2002) 67,125

159,167

Doe v. Wal-Mart Stores Inc 572 F 3d 677 (9th Cir 2009) .......................... 165, 249

Doe I v. Exxon Mobil Corporation, 393 F Supp 2d 20 (DDC 2005), aff'd 473 F 3d 345 (CADC 2007) $66,73,126$

Doe I v. Nestle USA, Inc738 F 3d 1048 (9th Cir 2013) 133,149

Dow Chem. v. Calderon, 422 F 3d 827, 832-3 (9th Cir 2005) ............................. 88

Dow Chemicals v. Castro Alfaro, 786 SW 2d 674 (S Ct Tex 1990) .... 60, 66, 87, 252

Du Daobin v. Cisco Systems Inc. Case 8:11-cv-01538-PJM, 24 Feb 2014 .... 74, 140 Enahoro v. Abubakar, 408 F 3d 877, 884-5 (7th Cir 2005), cert. denied, 546 US 1175 (2006) 
Erie Railroad Co v. Tompkins (1938) 304 US 64, 58 S Ct 817, 82 L Ed 1188 ....... 55, $96,98,110,166$

Estate of Manook v. Research Triangle Institute, International, F Supp 2d, 2010 WL 675264; (DDC 2010)

Estate of Manook v. Research Triangle Institute, International, 759 F Supp 2d 674 (EDNC 2010)

Estate of Rodriguez v. Drummond, 256 F Supp 2d 1250 (WD Al 2003) ............. 112

Filartiga v. Pena Irala 630 F 2d 876 (2d Cir 1980) .. 4, 41, 68, 91, 92, 93, 95, 96, 102, $103,119,253$

Flomo v. Firestone Natural Rubber Co 643 F 3d 1013, 1020-21 (7th Cir 2011) ... 47,

Flores v. SPCC, 253 F Supp 2d 510 (SDNY 2002), aff'd 343 F.3d 140 (2d Cir 2003) $62,63,152$

Flores v. Southern Peru Copper Corp 414 F 3d 233 (2d Cir 2003) .............. 117, 121

Ford ex rel. Estate of Ford v. Garcia, 289 F 2d 1283, 1286 (11th Cir 2002) ......... 111

Gallagher v. Neil Young Freedom Concert 49 F 3d 1442 (10th Circuit 1995) ...... 125

Galvis Mujica v. Occidental Petroleum Corporation 381 F Supp 2d 1164, (CD Cal 2005), 381 F Supp 2d 1134 (CD Cal 2005) .............................................. 76

Goodyear Dunlop Tires Operations, SA v. Brown 564 US (2011) $131 \mathrm{~S} \mathrm{Ct}$ 2846

Gulf Oil Corp v. Gilbert, 330 US 501, 508-9, 67 S Ct 839, 843-44, 91 L Ed 1055 (1947) $60,61,64,80$

Hamad v. Gates, 732 F 3d 990 (9th Cir 2013) 70

Hartford Fire Ins Co v. Cal, 509 US 764, 796 (1993) .................................. 44, 76

Hilton v. Guyot, 159 US 113, at 164, 16 S Ct 139, 40 L Ed 95 (1895) ................... 75

Homoki v.Conversion Servs Inc, 717 F 3d 388402 (5th Cir 2013) ..................... 105

Ibrahim v. Titan Corporation, 556 F Supp. 2d 1 (DDC. 2007) ................................69

Industria Panificadora, S.A. v. United States, 957 F 2d 886, 887 (DC Cir 1992) ... 68 Institute of Cetacean Research v. Sea Shepherd Conservation Soc, 860 F Supp 2d 1216 (WD Wash 2012) ......................................................................... 120

Institute of Cetacean Research v. Sea Shepherd Conservation Society, (2013) 725 F

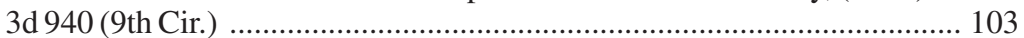

International Shoe Co. v. Washington, 326 US 310, 66 S Ct 154, 90 L Ed 95 (1945) 56

Jackson v. Metropolitan Edison Co, 419 US 345, 349, (1974) ........................... 125

Janko v. Gates 741 F 3d 136 (CA DC 2014) ....................................................... 70

Jogi v. Voges, 425 F 3d 367 (7th Cir 2005); 480 F 3d 822 (7th Cir 2007) .............. 92

John Doe I v. Unocal Corporation, 395 F 3d 932 (9th Cir 2002) ........... 72, 130, 132

John Roe v. Bridgestone Corporation, 492 F Supp 2d 988 (SD Indiana 2007) ... 121,

Julin v. Chiqita Brands International Inc, 690 F Supp 2d 1296 (SD Fla 2010) ..... 114 Kadic v. Karadzic 70 F 3d 232 (2d Cir 1995) .............................. 94, 122, 123, 126 Kasky v. Nike, 27 Cal 4th 939 (2002), cert. granted, 123 S Ct 817, and cert. dismissed, 539 US 654 (2003), 123 S Ct 2254 (2003)

Khulumani v. Barclay Nat. Bank Ltd, 504 F 3d 254 (2d Cir 2007) ...... 120, 121, 128, 130, 136, 142 
Kiobel v. Royal Dutch Petroleum Co 621 F 3d 111 (2d Cir 2010), 133 S Ct 1659

(2013); 2014 WL 2922840 (CA 4 (Va)) ..... 5, 42, 46, 47, 48, 56, 57, 59, 70, 77, 91, 95, 98-104, 105, 106, 107, 108, 109, 117, 143, 144, 145, 146, 147, 148, $149,166,172,196,251,252,254,255$

Klinghoffer v. SNC Achille Lauro, 937 F 2d 44,49 (2d Cir 1991) .......................... 73 Krishanthi v. Rajaratnam, 2010 WL 3429529 (DNJ) ............................... 122, 124

Laderer v. Dole Food Co. Inc No 12-09715 Class Action Complaint filed 13

November 2012 (US Dist Ct, CD Cal Los Angeles) ................................... 250

Licea v. Curacao Drydock Co, 584 F Supp 2d 1355, 1366 (SD Fla 2008) ............. 95

Licci rel. Licci v. Lebanese Canadian Bank 732 F 3d 161 (2d Cir 2013) ..... 148-149

Linde v. Arab Bank plc Case 1:04-cv-02799-BMC-VVP Document 1241 Filed 04/ 08/15. Slip Copy, 2015 WL 1565479 EDNY 2015 ........................... 115, 116

Liu v. Republic of China, 892 F 2d 1419 (9th Cir 1989) ......................................... 72

Marcos, In re Estate of 25 F 3d 1467 (CA 1994), 1475 .............................. 97, 103

Martinez v. Dow Chemicals, 219 F Supp 2d 719 (ED La 2002) ........................... 87

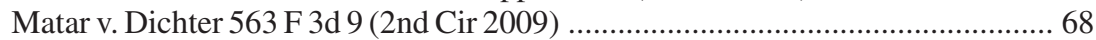

Mejia v. Dole Case No.BD40049, Los Angeles County Superior Court ........ 89, 152

Meshal v. Higgenbotham, 47 F Supp 3d 115 (DDC 2014) ............................... 112

Mohamad v. Palestinian Authority132 S Ct 1702 (2012) ......................... 112, 147

Mohamed v. Jeppesen Dataplan En Banc Order: 614 F 3d 1070 (9th Cir 2010) .... 70

Moose Lodge No. 107 v. Irvis, 407 US 163, 175, (1972) .................................... 125

Mora v. New York, 524 F.3d 183 (2d Cir.2008) .............................................. 122

Morrison v. Nat'l Australia Bank Ltd., 130 S Ct 2869, 2878 (2010) ...... 99, 100, 101

Mujica v. Airscan, 771 F 3d 580 (9th Cir 2014) ................................ 76, 109, 252

Mujica v. Occidental Petroleum Corp, 381 F Supp 2d 1164, 1179 n.13 (CD Cal 2005)

112,252

Murray v. The Charming Betsy, 6 US (2 Cranch) 64 (1804) ............................... 110

Mwani v. bin Laden 947 F Supp 2d 1 (DDC 2013), 2013 WL 2325166 ............... 107

Namba v. McCourt, 204 P 2d 569, 579 (Or 1949) …............................................. 78

National Association of Manufacturers v. Securities and Exchange Commission, 748 F 3d 359 (CA D 2014)

National Association of Manufacturers v. Securities and Exchange Commission,

2014 US App LEXIS 21753 (Nov. 18, 2014) .......................................... 223

Neumeier v. Kuehner, 31 NY 2d 121 (1972) ...................................................... 157

New York Central \& Hudson River Railroad v. US, 212 US 481 (1909) ................ 34

Ofori-Tenkorang v. American Int'l Group, Inc 460 F 3d 296, 298 (2d Cir 2006) ... 93

Osorio v. Dole Food Co 665 F. Supp 2d 1307 (SD Fla 2009) ................................ 88

Pacheco de Perez v. AT\&T Co, 139 F 3d 1368, (11th Cir 1998) ........................... 80

Papa v. United States, 281 F 3d 1004, (9th Cir 2002) ................................. 94, 112

Paquete Habana, The 175 US, 700, 20 S Ct 290 .............................................. 109

Patrickson v. Dole Food Co 251 F 3d 795, 803 (9th Cir 2001), aff 'd in part on other

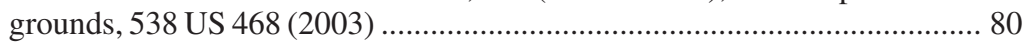

Piper Aircraft v. Reyno, 454 US 235 (1981) ............................................. 61, 64

Presbyterian Church of Sudan v. Talisman Energy 244 F Supp. 2d 289, 315-6 (SDNY 2003) $47,56,57,63,93,126,142,143$

Presbyterian Church of Sudan v. Talisman Energy 453 F Supp 2d 633 (SDNY 2006) 
Presbyterian Church of Sudan v. Talisman Energy, Inc, 582 F 3d 244 (2d Cir 2009)

Rivera v. Dole Food Co Los Angeles County Superior Court

Rivera v. Centro Medico de Turabo, Inc, 575 F 3d 10, 24 (1st Cir 2009) .............. 105

Romero v. Drummond 552 F 3d 1303, 1316 (11th Cir 2008) ............... 95, 142, 147

Rothstein v. UBS AG 08 F 3d 82 (2d Cir 2013) ................................................ 115

Rasul v. Myers, 563 F 3d 527 (CA DC 2009) …............................................. 120

Rylands v. Fletcher ............................................................................... 90

Sabbatino, 376 US 398, 84 S Ct 923 (S Ct 1964) ..................................... 72, 110

Sahu v. Union Carbide (Sahu I) WL 2422757 (S.D.N.Y., 2012) ................. 163, 164

Sahu v. Union Carbide (Sahu II) WL 3765556 (S.D.N.Y., 2014) ....................... 164

Saleh v. Titan Corporation Ltd 580 F 3d 1(CADC 2009) ...................................... 69

Saleh v. Titan Corp, 580 F 3d 1 (DC Cir 2010) ....................................................... 106

Sanchez-Espinoza v. Reagan, 770 F 2d 202, 207 (DC Cir 1985) .................. 69, 106

Sarei v. Rio Tinto, 221 F Supp 2d 1116, 1126 (CD Cal 2002); F 3d 2006 WL 2242146 (9th Cir Aug 7, 2006) ................. 1, 3, 56, 63, 64, 66, 72, 76, 120, 125

Sarei v. Rio Tinto, 487 F 3d 1193 (9th Cir 2007) .............................................. 150

Sarei v. Rio Tinto, 671 F 3d 736 (9th Cir 2011) .............. 98, 140, 141, 142, 145, 146

S.E.C. v. Obus, 693 F 3d 276, 292 (2d Cir.2012) ............................................. 105

Serra v. Lappin, 600 F 3d 1191 (2010) ......................................................... 110

Sexual Minorities Uganda v. Lively 960 F Supp 2d 304 (D Mass 2013) .............. 106

Seymour v. Hull \& Moreland Engineering 605 F 2d. 1105, 1111 (9th Cir

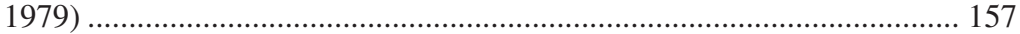

Siderman de Blake v. Republic of Argentina, 965 F 2d 699 (9th Cir 1992) ...... 45, 67

Sinaltrainal v. Coca Cola, 256 F Supp 2d 1345 (SD Fla 2003) ................... 112, 165

Sinaltrainal v. Coca Cola, 578 F 3d 1252, 1261 (11th Cir 2009) ................... 93, 123

Sinochem Int'l Co Ltd v. Malaysia Int'1 Shipping Corp, 549 US 422, 429 (2007)

Sosa v. Alvarez Machain 542 US 692, 124 S Ct 2739 ...... 95-98, 100, 103, 106, 110, $117,120,123,128,129,130,131,141,144,146,151$

South African Apartheid Litigation 346 F Supp.2d 538 (SDNY 2004) ........ 130, 131

South African Apartheid Litigation, re, 617 F Supp 2d 228, 256 (SD NY 2009) ..... 67, 74, 94, 97, 120, 128, 130, 132, 133, 137, 138, 139, 150, 151, 156,

South African Apartheid Litigation, In re No. 02 MDL 1499, 2013 WL 6813877,

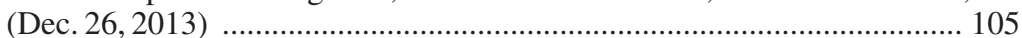

South African Apartheid Litigation, In re 15 F.Supp.3d 454 (SDNY 2014) ....... 149

Speelman v. United States, 461 F Supp 2d 71, 73 (DDC 2006) ........................... 92

Strauss v. Credit Lyonnais, S.A., 925 F Supp 2d 414 (EDNY 2013) ................... 115

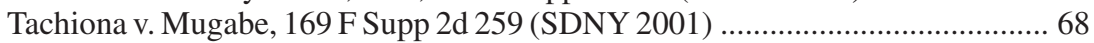

Tachiona v. Mugabe, (2002) 234 F Supp 2d 401 ............................................... 95

Talbot v. Janson, 3 US (3 Dall) 133, 156-8, 1 L Ed 540, 3 Dall 133 (1795) .......... 131

Tel-Oren v. Libyan Arab Republic, 726 F 2d 774, (CADC 1984) ............ 96, 97, 253

Tellez v. Dole Food Co. Los Angeles County Superior Court ............................. 89

Terrorist Attacks on September 11, 2001, In re, 714 F 3d 659 (2d Cir 2013) ...... 112,

Torres v. Southern Peru Corporation 113 F 3d 540, 542-3(5th Cir 1997) $\begin{array}{r}122 \\ 79\end{array}$ 
Union Carbide Gas Plant Disaster, re, 634 F Supp 842 (SDNY 1986), aff'd. 809 F

2d. 195 (2d Cir 1987) $4,62,64,65,66,152$

Unocal (2002) see John Doe I v. Unocal Corporation, 395 F 3d 932 (9th Cir 2002)

US v. Bestfoods, 524 US 51, 118 S Ct 1876 (1998) ........................... 155, 157, 161

United States v. Price, 383 US 787, 794, (1966) .............................................. 125

United States v. Reynolds 345 US 1, 11 (1953) ................................................. 70

Vietnam Ass'n for Victims of Agent Orange v. Dow Chem. Co, 517 F 3d 104, 115

(2d Cir 2008)

Wiwa v. Royal Dutch Petroleum Co 226 F $3 d 88$ (2d Cir 2000) ..... 57, 61, 64, 65, 93 ,

Wiwa (2009), 626 F Supp 2d 377 (SDNY 2009)

Wiwa v. Royal Dutch Petroleum Co, No. 96 Civ. 8386, 2002 WL 319887, at *7-9

(S.D.N.Y. Feb. 28, 2002)

Wultz v. Islamic Republic of Iran, 755 F Supp 2d 1 (DDC 2010) ....................... 114

XE Services, re 665 F Supp 2d 569 (ED Va 2009) ................................... 123, 147

Xuncax v. Gramajo 886 F Supp 162, 182-4 (Mass DC 1995) ....................... 95, 110

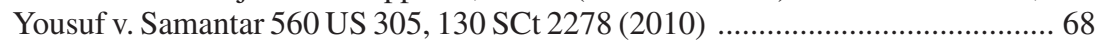

Zerilli- Edelglass v. New York City Transit Auth, 333 F 3d 74, 80-81 (2d Cir 2003)

Zschernig v. Miller, 389 US 429, 432, 88 SCt 664, 19 L Ed 2d 683 (1968) 79

\section{INTERNATIONAL CASES}

\section{African Commission}

Commission Nationale des Droits de l'Homme et des Libertés v. Chad, African Commission Communication No 74/92 (1995) ......................................... 8

SERAC case, African Commission, Communication No 155/96 (2001) ................ 8

\section{CAFCA Arbitration}

Pacific Rim, December 2007 52

\section{Court of Justice of the Economic Community of West African States (ECOWAS)}

SERAP v. Federal Republic of Nigeria, Judgment $\mathrm{N}^{\circ}$ ECW/CCJ/JUD/18/12 8

\section{European Court of Justice}

Arblade (Case C-369/96) [1999] ECR I-8453

Owusu v. Jackson (Case C-281/02) [2005] ECR I-1383 ..... 43, 174, 198

\section{European Court of Human Rights}

Al Adsani v. UK, (2002) 34 EHRR 11 $45,68,179,199$

Jones and Others v. United Kingdom (Applications nos. 34356/06 and 40528/06) Judgment 14 January 2014 $45,68,201$ 
Osman v. UK, [1988] EHRR 101 8

Van Anraat v. the Netherlands, Application no. 65389/09, 20 July 2010 38

\section{Inter-American Court of Human Rights}

Velásquez Rodríguez v. Honduras, [1988] Inter-Am Court HR (ser C) No 4 8

\section{International Court of Justice (ICJ)}

Advisory Opinion on the Legal Consequences on the Construction of a Wall in the Occupied Palestinian Territory, (2004) 43 ILM 1009

Armed Activities on the Territory of the Congo (Democratic Republic of the Congo v. Uganda), (Merits) (2006) 45 ILM 271

Arrest Warrant case: Case concerning Arrest Warrant of 1 I April 2000 (Democratic Republic of the Congo v. Belgium), Judgment, ICJ Reports 2002, p. 3 (February 14, 2002) ............................................................... 30, 32, 201

Barcelona Traction, Light and Power Co Ltd (Second Phase) (Belgium v. Spain), [1970] ICJ Reports 3, 42

Bosnia and Herzogovina v. Serbia and Montenegro (26 February 2007) ... 8, 10, 146

Certain Phosphate Lands in Nauru (Nauru v. Australia), Preliminary Objections, Judgment (sep. op. Shahabuddeen), ICJR (1992), 240, 290

Jurisdictional Immunities of the State (Germany v. Italy) Judgment (Feb. 3, 2012)

Nicaragua v. US (Case concerning Military and Paramilitary Activities In and Against Nicaragua) [1986] ICJ Rep 14 10,206

Nottebohm Case, (Liech. v. Guatamala), 1955 ICJ 4, 23 (Apr. 6) ..... 44

\section{International Criminal Court (ICC)}

Prosecutor v. Thomas Lubanga Dyilo, Case No. ICC/01/04-01/06, Pre-Trial Chamber Decision on the Confirmation of Charges (Jan. 29, 2007) ... 138, 140

Situation in the Republic of Kenya, Case No. ICC-01/09, Decision Pursuant to Article 15 of the Rome Statute on the Authorization of an Investigation into the Situation in the Republic of Kenya (Mar. 31, 2010)

\section{International Criminal Tribunal for the former Yugoslavia (ICTY)}

Prosecutor v. Furundzija, IT-95-17/1 (Dec. 10, 1998) ..... 15, 22, 127, 130, 132, 133,

Prosecutor v. Krstic IT-98-33-A (19 April 2004)

Prosecutor v. Nikola Šainović IT-05-87-A (Appeals Chamber), 23 January 2014 $22,133,135,141$

Prosecutor v. Perišic IT-04-81-A (Feb. 28, 2013) $22,133,140$

Prosecutor v. Tadicć IT-94-1-A (15 July 1999) $10,15,21,129$

Prosecutor v. Vasiljevic, IT-98-32-A, (App. Chamber, Feb. 25, 2004) 132-133

\section{International Criminal Tribunal for Rwanda (ICTR)}

Prosecutor v. Akayesu, ICTR-96-4-T (Trial Chamber), September 2, 1998 .......... 17

Prosecutor v. Ntuyahaga, ICTR-90-40-T, Mar. 18, 1999 


\section{NAFTA Arbitration}

Lone Pine, November 2012

\section{Special Court for Sierra Leone (SCSL)}

Prosecutor v. Taylor SCSL-03-01-A (10766-11114 Sept. 26, 2013) ...... 22, 31, 133,

Case Concerning the Arrest Warrant of 11 April 2000 (Democratic Republic of the Congo v. Belgium) February 14, 2002

\section{International Military Tribunal (IMT) Nuremberg}

United States v. Krauch et al. (IG Farben Case), Trials of War Criminals Before the Nuremberg Military Tribunals Under Control Council No. 10, Vol. VIII (1952)

United States v. Krupp, 9 Trials of War Criminals Before the Nuremberg Military

Tribunals Under Control Council Law No. 10, 1327, 1352-53 (1950)

United States v. Von Weizsacker (The Ministries Case), 14 Trials of War Criminals Before the Nuremberg Military Tribunals, 622, 851-2 (1950) ... 134, 135, 137,

\section{British Military Court, Hamburg}

Trial of Bruno Tesch and Two Others (The Zyklon B Case) 1 Law Reports of Trials of War Criminals 93-103 (1947)

\section{Permanent Court of Arbitration}

Chevron case 85,86

Permanent Court of International Justice

\section{NATIONAL LEGISLATION}

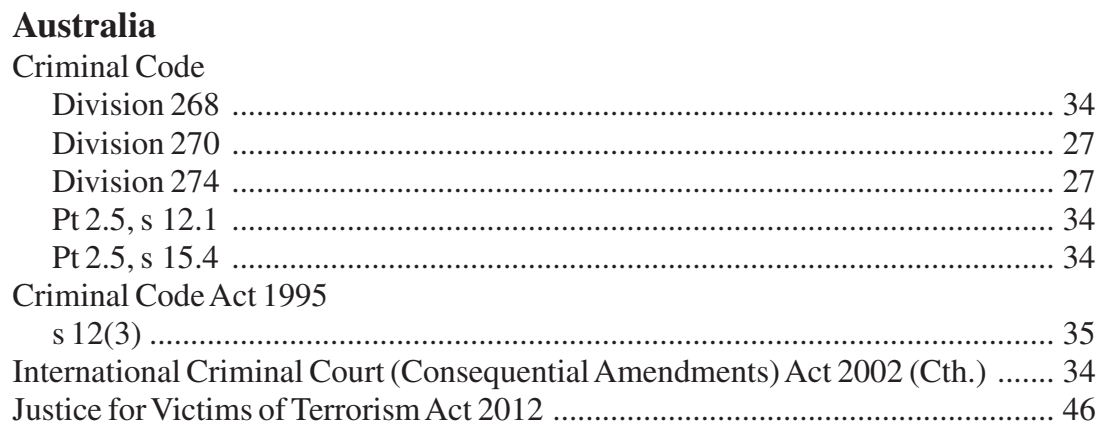




\section{Belgium}

Act on the Punishment of Grave Breaches of International Humanitarian Law, 1993

(amended 1999)

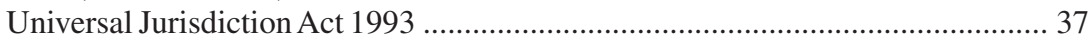

\section{Canada}

Charter of Rights and Freedoms

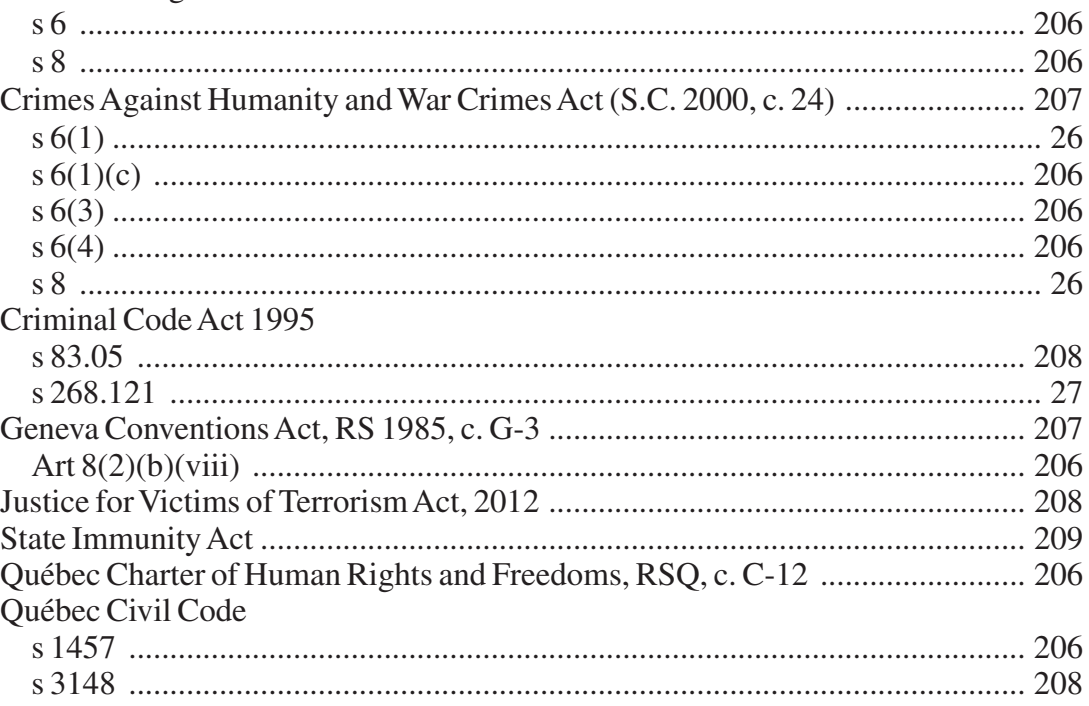

\section{Ecuador}

Constitution

Art 19(2)

Environmental Management Act 1999

Corporations Law (Ley de Compañías)

Art 17

\section{France}

Civil Code

Art 1382

Code of Criminal Procedure

\section{Germany}

Code of Crimes Against International Law 2002, 42 ILM (2003) 995

Art 1

\section{Ireland}

Constitution

Art 15, s 2 


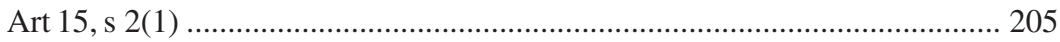

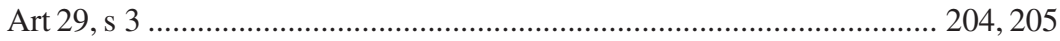

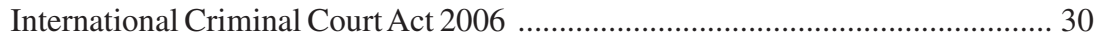

\section{Netherlands}

International Crimes Act 2003 ................................................................. 30, 39

Penal Code

Art 48

\section{New Zealand}

International Crimes and International Criminal Court Act 2000

\section{Nicaragua}

Special Law 364, 2000 ....................................................................... 8 87, 88

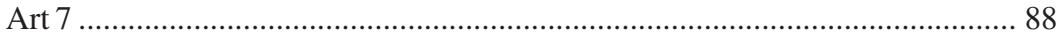

\section{Nigeria}

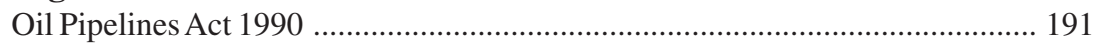

\section{Papua New Guinea}

OK Tedi Mine Continuation (Ninth Supplemental) Act 2001

\section{South Africa}

Implementation of the Rome Statute of the International Criminal Court Act 2002

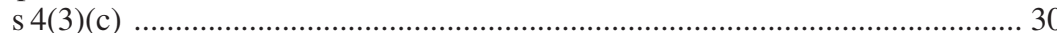

Mining and Petroleum Resources Development Act (MPRDA) ................. 53, 231

\section{Spain}

Universal Jurisdiction Law ....................................................................... 25, 26

\section{United Kingdom}

Asylum and Immigration (Treatment of Claimants, etc,) Act 2004

s 4

Companies Act 1985

s $694 \mathrm{~A}$

s 695

Companies Act 2006

Coroners and Justice Act 2001

s $70(3)$

Coroners and Justice Act 2009

s 71

Corporate Manslaughter and Corporate Homicide Act 2007

s $1(1)$

s $1(3)$ 
Criminal Justice Act 1988

s 134

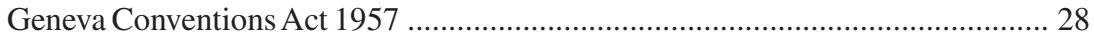

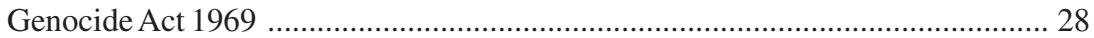

Health and Safety at Work Act 1974 ............................................................... 35

Insolvency Act

s 423

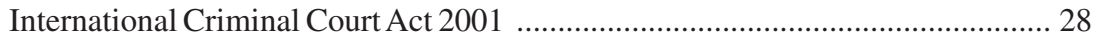

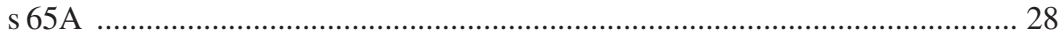

International Crimes Act 2001

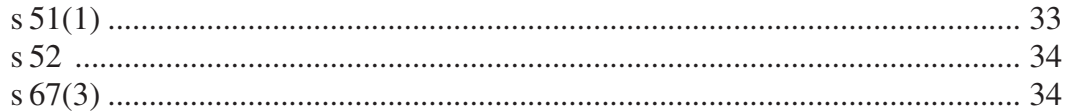

Interpretation Act 1978

Sch

Legal Aid, Sentencing and Punishment of Offenders Act 2012 ......................... 246

Modern Slavery Act 2015 .......................................................................... 29

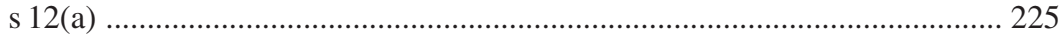

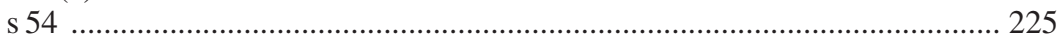

Private International Law (Miscellaneous Provisions) Act 1995

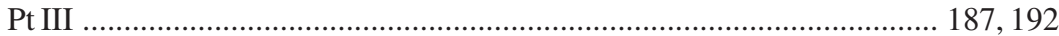

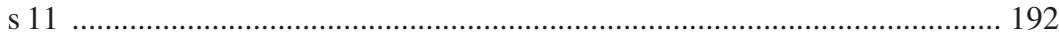

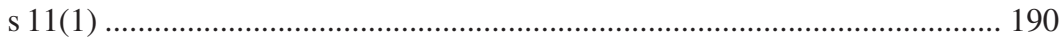

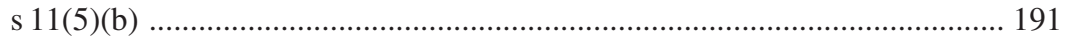

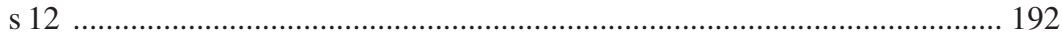

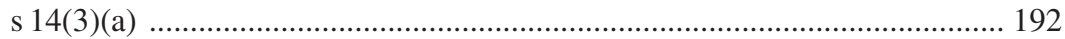

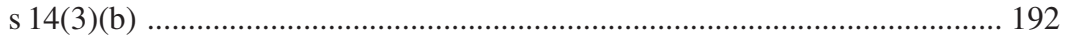

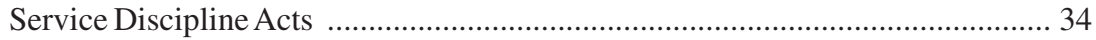

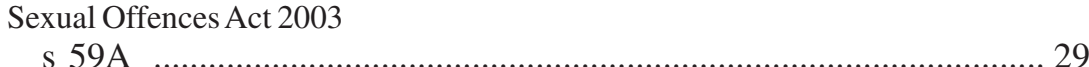

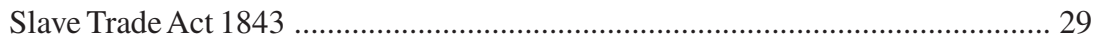

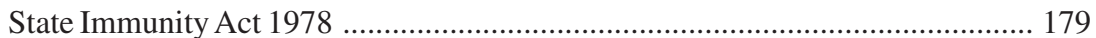

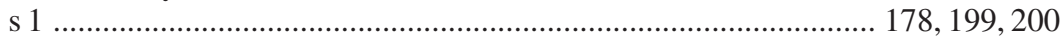

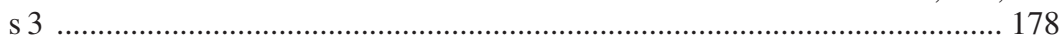

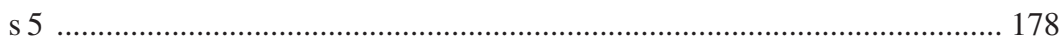

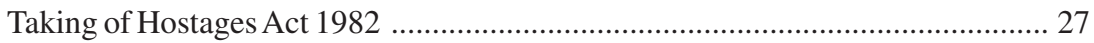

War Crimes Act 1991 ............................................................................ 28

Companies Act 2006 (Strategic Report and Directors' Report) Regulations 2013

(Strategic Report Regulations) ............................................................... 224

Criminal Procedure Rules (CPR)

r $6.2(2)$


r 6.36

PD 6B, s 3.1

Reports on Payments to Governments Regulations 2014

\section{United States of America}

Constitution

Art III $59,78,152$

Art III(2) 54

First Amendment 223,249

Fifth Amendment 55

Fourteenth Amendment 55

Alien Torts Statute 1789 (ATS), 28 USC s. 1350 ...... 4, 41, 45, 46, 55, 60, 61, 66, 74, $75,78,79,91-116,120,123,124,125,126,127,128,129,130,131,132,141$, $142,143,144,145,146,147,148,149,150,151,152,153,155,156,167,170$, $172,177,196,211,238,251,252,253,254,255$

s 1367 78

s 1441 79

s 1441(b)(2) 79

s 1441 (c) 78

s 1441(f)

Anti-Terrorism Act 1992 (ATA), 18 US Code $\$ 2333$. $114,115,116$

Anti-Terrorism and Effective Death Penalty Act of 1996 113

Civil Rights Act, 1871 124,125

Comprehensive Environmental Response, Compensation and Liability Act of 1980 (CERCLA) 155,162 s 107(a)(2) 161

Dodd-Frank Act s 1502 222 s 1504 223

Federal Tort Claims Act 1948 (FTCA) $68,69,70$

Foreign Corrupt Practices Act .84

Foreign Sovereign Immunity Act …………............................................... 95

28 USC section 1330, sections 1602-11 ..................................................... 45

Genocide Accountability Act 2007, Pub L No. 110-151, 121 Stat 1821 (codified as amended at 18 USC $\S 1091$ (2006 \& Supp. IV 2010) ................................. 30

Mandatory Victims Restitution Act of 1996 ........................................... 112, 113

Military Commissions Act (MCA) ............................................................. 70

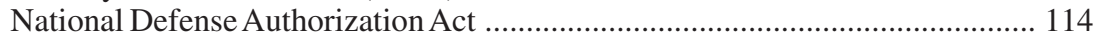

Proxmire Act 1988 ..................................................................................... 30

Racketeer Influenced and Corrupt Organisations Act (RICO) ........................... 84

Torture Victims Protection Act 1991 (TVPA) .............. 61, 65, 91, 94, 110, 111-112

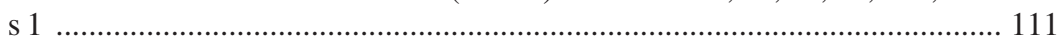

Trafficking Victims Protection Act of 2000 .................................................. 116

Trafficking Victims Protection Reauthorisation Act 2003 (TVPRA 2003) .......... 116

Uniform Foreign-Country Money Judgments Recognition Act 2005 .................. 81

Uniform Foreign Money Judgment Recognition Act 1962 ............................... 81

Federal Rules of Civil Procedure 
r 8(a)(2)

Restatement (Second) of Agency

$14 \mathrm{M}$

Restatement (Second) of Conflict of Laws

s 145

Restatement (Second) of Torts

s 876

Restatement (Third) of Foreign Relations

s 402

s 402(2)

44

s 403

103

s 403(2)

75

s 404

$30,103,120$

California, Civil Proc Code Ann $\$ 410.10$........................................................ 58

California, Consumer Legal Remedies Act (Civ Code s 1750 et. seq) .................. 250

California, False Advertising Law ................................................................... 249

California, Transparency in Supply Chains Act 2010 ......................................... 224

California, Unfair Competition Law (Cal Bus \& Prof Code s 17200 et seq) ...... 249,

250

Florida Uniform Foreign Money Judgment Recognition Act ....................... 81, 88

New York Recognition of Foreign Country Money Judgments Act ..................... 83

\section{EU LEGISLATION}

Brussels Regulation (EC) No. 44/2001 …................................................... 172

Art 2

$43,173,174$

Art 5.3

173

Art 6(1)

43,173

Art 60.1

173

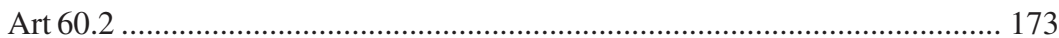

Regulation (EU) No. 1215/2012 on jurisdiction and recognition an enforcement of judgments in civil and commercial matters (recast) (Judgments

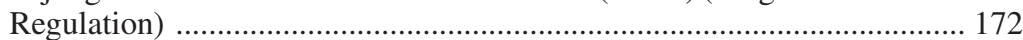

Art 4

173,174

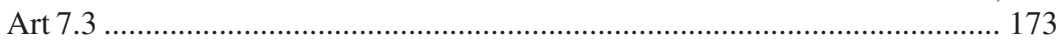

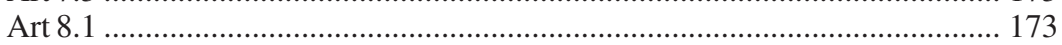

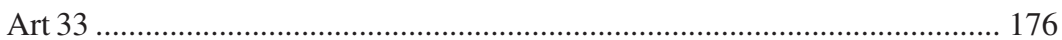

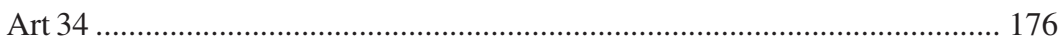

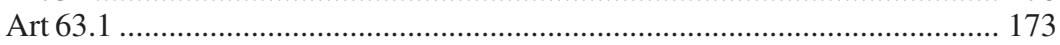

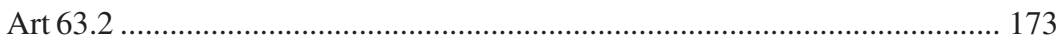

Regulation (EC) No 864/2007 on the law applicable to non-contractual obligations

(Rome II) 190,193 


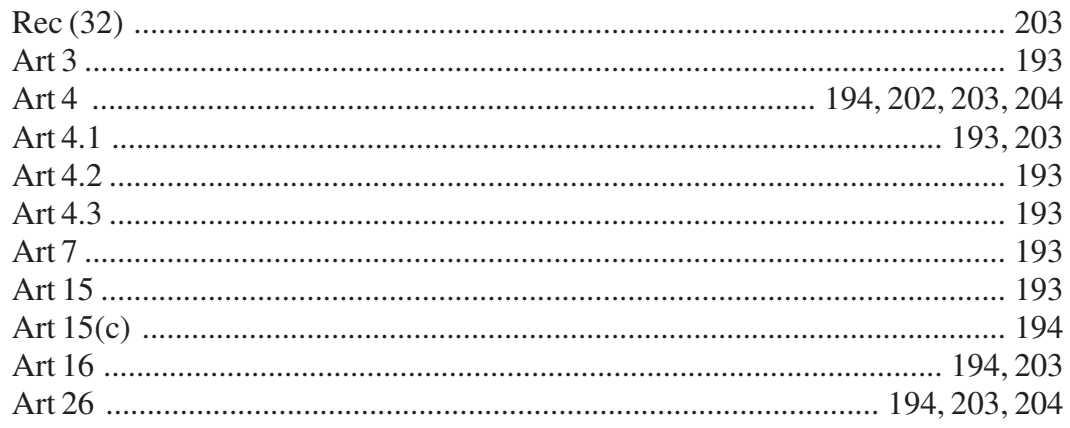

Accounting Directive, Dir 2013/34/EU (amending Directive 2006/43/EC of the European Parliament and of the Council and repealing Council Directives 78/660/EEC and 83/349/EEC) ........................................................... 224-225

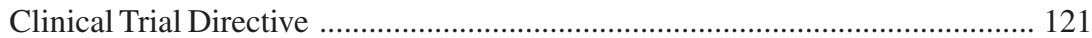

Directive 2014/95/EU on disclosure of non-financial and diversity information by certain large undertakings .................................................................. 224

\section{TREATIES AND INTERNATIONAL INSTRUMENTS}

African Charter on Human and Peoples' Rights (ACHPR) …........................... 8, 9

American Convention on Human Rights (1969) …............................................ 119

American Declaration of the Rights and Duties of Man (1948) ........................ 118

Australia-Hong Kong Bilateral Investment Treaty ........................................... 51

Central American Free Trade Agreement (CAFTA) 2004 ...................... 49, 51, 52

Comprehensive Economic and Trade Agreement (CETA) (EU/Canada) .............. 49

Convention on Human Rights and Medicine, Council of Europe, 1997 Art 5

Convention on the Prevention and Punishment of the Crime of Genocide, 1948 (78

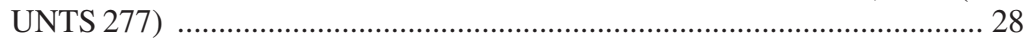

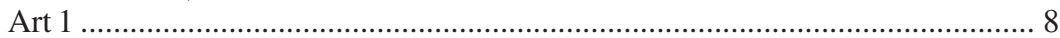

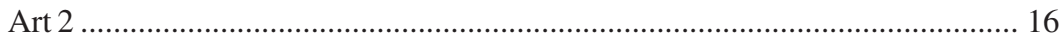

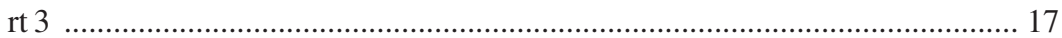

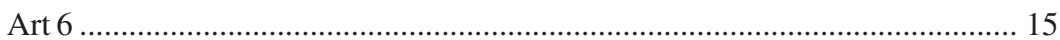

Convention on the Rights of the Child (CRC), 1989 ............................... 8, 9, 20

Convention on the Safety of United Nations and Associated Personnel, 1994 ...... 20

Declaration of Helsinki, World Medical Association, 1964 (1996) ..................... 121

Draft Articles on Responsibility of States for Internationally Wrongful Acts .... 9, 10

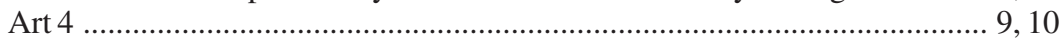

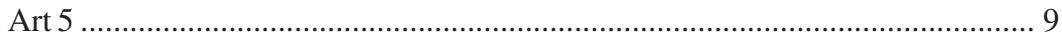

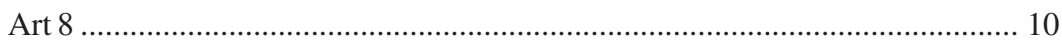

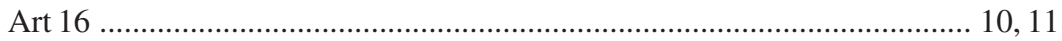

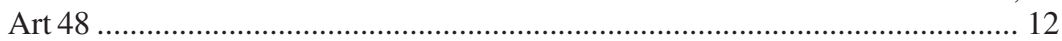

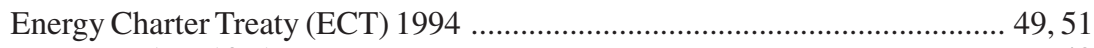

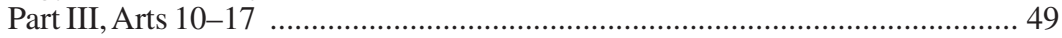

European Convention on Human Rights ..................................................... 8 
Art 4

Art 6

Geneva Conventions (I) for the Amelioration of the Condition of the Wounded and

Sick in Armed Forces in the Field; (II) for the Amelioration of the Condition of Wounded, Sick and Shipwrecked Members of Armed Forces at Sea; (III) relative to the Treatment of Prisoners of War; (IV) relative to the Protection of Civilian Persons in Time of War, 1949 20,21 Art 3 $20,28,147$

Art 49 210

Art 49(6) 206

Art 53 210

Additional Protocol I 20

Additional Protocol II 20

Geneva Protocol, 1925 20

Hague Convention 1907

Art 23g

Art 46(2)

Hague Convention for the Protection of Cultural Property in the Event of Armed

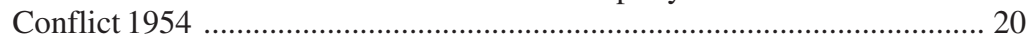

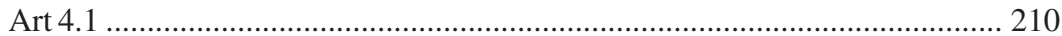

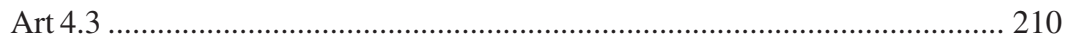

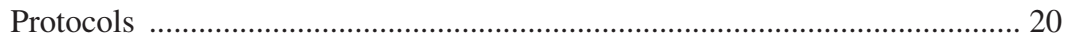

Hague Declaration, 1899 ................................................................................... 20

Hague Regulations 1907 annexed to the Hague Convention No. IV ................ 20, 21

International Convention Against the Taking of Hostages, 1979 ......................... 28

International Covenant on Civil and Political Rights (ICCPR), 999 UNTS 171 . 8, 9, $13,118,121,122,201,233$

Art 2

Art 2(2)

Art 2(3)

Art 7

International Covenant on Economic, Social and Cultural Rights (ICESCR), 999

UNTS 3

$7,8,13,14,119,233$

International Criminal Court Act 2001, explanatory notes 23

International Labour Organization (ILO) core conventions 13,226

International Labour Organization Declaration on Fundamental Principles and Rights at Work 213,234

International Law Commission, Draft Articles on Responsibility of States for Internationally Wrongful Acts

Art 4

Art 5

Art 8

Art 16

Art 48

International Maritime Organisation Collision Regulations …........................ 192

Nuremburg Charter of the International Military Tribunal, 8 UNTS 279, August 8, 1945 
$\operatorname{Art} 6(2)(\mathrm{c})$

Nuremburg Code

121,122

Rio Declaration on Environment and Development (1992)

118,213

Rome Statute, 1998 (International Criminal Court)

4, 17, 24, 25, 27, 28, 29, 30,

$132,137,143$

Art 5

Art 7

$15,18,19$

$\operatorname{Art} 7(2)(a)$

Art 8

Art 8(2)(a)

Art 8(2)(a)(iv)

Art 8(2)(b)

Art 8(2)(b)(viii)

Art 8(2)(b)(xvi)

Art 8(2)(d)

Art 8(2)(e)

Art 8(2)(e)(v)

Art 8(e)(xii)

Art 8(2)(f)

Art 10

Art 17

Art 25 .

Art 25(1)

Art 25(3)

23, 136

Art 25(3)(a)

Art 25(3)(c)

$135,136,139$

Art 25(3)(d)

Art 25(c)

137,138

Art 25(d)

23, 136

Art 27 31

Art 30

136,140

Art 30(1) 23

Art 30(2)

$23,138,139$

Art 30(2)(b)

139,140

Art 86

Art 121

Art 123

Statute for the International Criminal Tribunal for the Former Yugoslavia, 1993

Art 5

Statute for the International Criminal Tribunal for Rwanda, 1994 ................ 17, 20

Art 3

Statute of the International Court of Justice

Art 38

Statute of the Special Court for Sierra Leone .................................................. 20

Tokyo Charter 1946 (International Military Tribunal for the Far East) ..................... 18

Transatlantic Trade and Investment Partnership (TTIP) 
UN Charter

Art 55c 78

Art 56

UN Convention Against Corruption ……...................................................... 213

UN Convention Against Torture and Other Cruel, Inhuman or Degrading Treatment or Punishment (UNCAT), UNGA Res. 39/46, 39 UN GAOR Supp. (No. 51) UN Doc. A/39/51 (1984) ................................ 24, 27, 29, 178, 197, 199, 201

Art 4

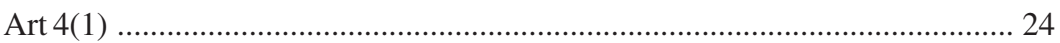

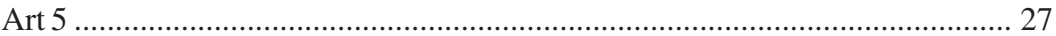

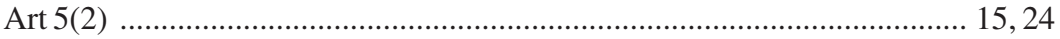

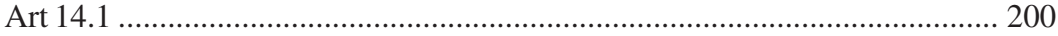

UN Convention on the Rights of the Child (1989) ........................................... 119

UN Convention on the Law of the Sea (UNCLOS) .......................................... 72

Universal Declaration of Human Rights, 1948 ............................ 13, 14, 119, 233

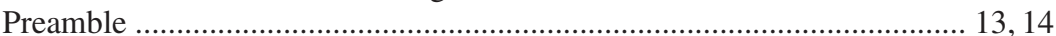

Universal Declaration on Bioethics and Human Rights, UNESCO, 2005 ........... 121

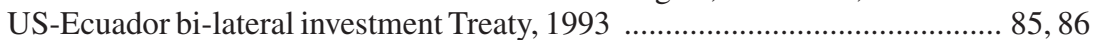

Vienna Convention on Consular Relations, Apr. 24, 1963, 21 UST 77, TIAS No. 6820

\section{VOLUNTARY CODES}

Accord on Fire and Building Safety in Bangladesh ....................................... 221

Alliance for Bangladesh Worker Safety ...................................................... 222

Extractive Industries Transparency Initiative (EITI), 2002 .......................... 219

International Labour Organisation (ILO) Declaration of Principles concerning

Multinational Enterprises and Social Policy (MNE Declaration) ....... 226, 247

ILO Declaration on Fundamental Principles and Rights at Work, 1998 .............. 226

International Code of Conduct for Private Security Service Providers (ICOC) ... 245

Kimberley Process Certification Scheme, 2003 ............................................... 222

OECD Common Approaches, 2012 .............................................................. 245

OECD Guidelines for Multinational Enterprises, 2011 ... 6, 217-219, 232, 239, 247

UN Commission on Transnational Corporations Code of Conduct ................... 226

UN Draft Norms on the Responsibilities of Transnational Corporations and Other

Business Enterprises with Regard to Human Rights (Draft Norms), 2003

UN Global Compact, 2000 ..................................... 5, 212-214, 247, 248, 249

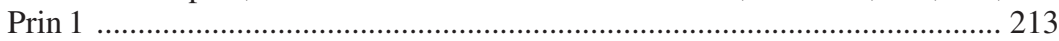

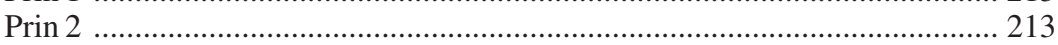

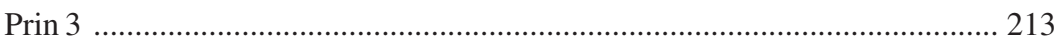

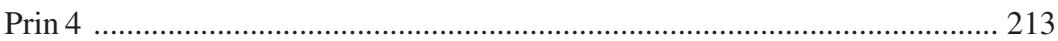

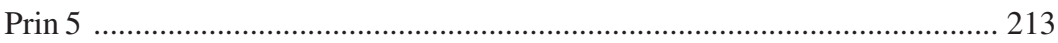

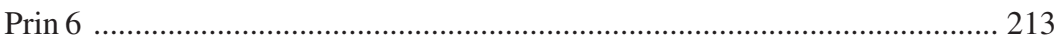

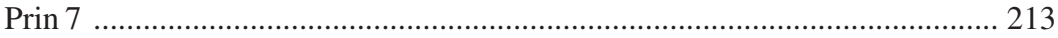

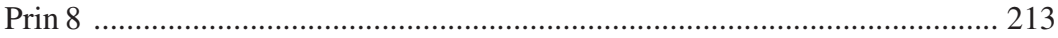


Prin 9

Prin 10

UN Guiding Principles on Business and Human Rights, 2011

6, 213, 227-248,

GP 1

GP 2

$228,229,239$

GP 3

229,256

GP 4

GP 5

230

GP 6

230

GP 7

230

GP 8

231

GP 9

231

GP 10

231

GP 11

232

GP 12

234

GP 13(a)

233

GP 13(b)

234

GP 14

234

GP 15

234

GP 16

235

GP 17

235

GP 18

235

GP 19

236

GP 20

GP 21

GP 22

GP 23

GP 24

GP 25

GP 26

GP 27

GP 28

GP 29

GP 30

GP 31

$233,234,236$
$\ldots \ldots \ldots \ldots . . . . .237$

237

$233,234,236,237,241$

238,242

238

$228,239,240$

$233,239,255$

240

241

241

241

238, 241

UN Working Group (UNWG) Guidance on National Action Plans on Business and

Human Rights, 2014 245

Voluntary Principles on Security and Human Rights, 2000 $5-6,214-216$ 